\title{
CAUCHY-DIRICHLET PROBLEM FOR THE NONLINEAR DEGENERATE PARABOLIC EQUATIONS
}

ISMAIL KOMBE

Received 30 May 2004

We will investigate the nonexistence of positive solutions for the following nonlinear parabolic partial differential equation: $\partial u / \partial t=\mathscr{L} u+V(w) u^{p-1}$ in $\Omega \times(0, T), 1<p<$ $2, u(w, 0)=u_{0}(w) \geq 0$ in $\Omega, u(w, t)=0$ on $\partial \Omega \times(0, T)$ where $\mathscr{L}$ is the subelliptic $p$ Laplacian and $V \in L_{\mathrm{loc}}^{1}(\Omega)$.

\section{Introduction}

This paper deals with the nonexistence of positive solutions to the following nonlinear parabolic equation:

$$
\begin{array}{ll}
\frac{\partial u}{\partial t}=\mathscr{L} u+V(w) u^{p-1} & \text { in } \Omega \times(0, T), 1<p<2, \\
u(w, 0)=u_{0}(w) \geq 0 & \text { in } \Omega, \\
u(w, t)=0 & \text { on } \partial \Omega \times(0, T),
\end{array}
$$

where $\Omega$ is a Carnot-Carathéodory metric ball in $\mathbb{R}^{2 n+1}$ and $V \in L_{\text {loc }}^{1}(\Omega)$. The nonlinear operator $\mathscr{L}$ is the subelliptic $p$-Laplacian:

$$
\mathscr{L} u=\sum_{j=1}^{2 n} X_{j}\left(|X u|^{p-2} X_{j} u\right),
$$

where

$$
X_{j}=\frac{\partial}{\partial x_{j}}+2 k y_{j}|z|^{2 k-2} \frac{\partial}{\partial l}, \quad X_{n+j}=\frac{\partial}{\partial y_{j}}-2 k x_{j}|z|^{2 k-2} \frac{\partial}{\partial l}, \quad j=1, \ldots, n .
$$

are the smooth vector fields and satisfy Hörmander condition [25] for any $k \in \mathbb{N}$. Here $X u=\left(X_{1} u, \ldots, X_{2 n} u\right)$ is the subelliptic gradient of a function $u$. Observe that

$$
\mathscr{L} u=\sum_{j=1}^{2 n} X_{j}\left(|X u|^{p-2} X_{j} u\right)=0
$$


is the Euler-Lagrange equation of the variational integral

$$
J_{p}(u)=\frac{1}{p} \int|X u|^{p}
$$

where

$$
|X u|=\left[\sum_{j=1}^{2 n}\left(X_{j} u\right)^{2}\right]^{p / 2}, \quad p>1
$$

The subelliptic $p$-Laplacian

$$
L u=\sum_{i=1}^{m} X_{i}\left(|X u|^{p-2} X_{i} u\right)=0
$$

was studied by Capogna et al. [7] for more general systems of $C^{\infty}$ vector fields which satisfy Hörmander condition. They established sharp Sobolev embedding associated to the functional $J_{p}$ and the Harnack inequality for positive solutions of (1.7). The fundamental solution of the subelliptic $p$-Laplacian (1.2) at the origin has been found by Zhang and Niu [36]. In that paper they established Hardy-type inequalities and Pohozaev-type identities associated with vector fields (1.3).

If $p=2$ then subelliptic $p$-Laplacian (1.2) reduces to sub-Laplacian

$$
\Delta_{k}=\sum_{j=1}^{2 n} X_{j}^{2}
$$

which arise in a diverse area of mathematics including boundary value problems in several complex variables, harmonic analysis, quantum mechanics of anharmonic oscillators and electromagnetic fields. The hypoellipticity of $\Delta_{k}$ follows from the famous paper of Hörmander [25]. The fundamental solution of $\Delta_{k}$ has been studied extensively by Greiner [24] and Beals et al. [2, 3, 4]. In a recent paper, Kombe [32] studied nonlinear parabolic equations and found sharp Hardy-type inequality associated with $\Delta_{k}$.

Note that if $k=1$ then $\Delta_{k}$ becomes Kohn-Laplacian $\Delta_{\mathbb{\square} n}$ on the Heisenberg group $\mathbb{\square}^{n}$ and today there is a large literature about partial differential equations on the Heisenberg group (see $[9,10,12,13,16,17,21,22,23,26,27,28]$ and references therein).

Problem (1.1) has been studied on the Euclidean space and Heisenberg group by Goldstein and Kombe $[20,21]$ and for the nonsmooth vector fields by Kombe [30, 31]. It turn outs that nonexistence of positive solutions that kind of problems largely depends on the size of bottom of the normalized $p$-energy forms

$$
E(V):=\frac{\int_{\Omega}|X \phi|^{p} d w-\int_{\Omega} V|\phi|^{p} d w}{\int_{\Omega}|\phi|^{p} d w},
$$

where $\phi \in C_{c}^{\infty}(\Omega)$ and $V \in L_{\text {loc }}^{1}(\Omega)$. Clearly, in the case $k=1$, our results recovers the results of Goldstein and Kombe [21]. The following is the main result of this paper. 
Theorem 1.1. Let $(4 n+4 k) /(2 n+2 k+1) \leq p<2$ and $V \in L_{\mathrm{loc}}^{1}(\Omega \backslash \mathscr{K})$, where $\mathcal{K}$ is a closed Lebesgue null subset of $\Omega$. If

$$
E_{\text {inf }}((1-\epsilon) V):=\inf _{0 \neq \phi \in C_{c}^{\infty}(\Omega \backslash \mathscr{K})} \frac{\int_{\Omega}|X \phi|^{p} d w-\int_{\Omega}(1-\epsilon) V|\phi|^{p} d w}{\int_{\Omega}|\phi|^{p} d w}=-\infty
$$

for some $\epsilon>0$, then the problem (1.1) has no general positive local solution off of $\mathscr{K}$.

The outline of this paper is the following. In Section 2, we introduce notations, spherical transformation, basic lemma and Hardy-type inequality. In Section 3, we prove our main Theorem and two corollary.

\section{Preliminary and notations}

The generic point is $w=(z, l)=(x, y, l) \in \mathbb{R}^{2 n+1}$. For $w_{0} \in \mathbb{R}^{2 n+1}$ and $r>0, \Omega=B_{d_{c}}\left(w_{0}, r\right)=$ $\left\{w \in \mathbb{R}^{2 n+1} \mid d_{c}\left(w, w_{0}\right)<r\right\}$ denotes the $d_{c}$-metric ball in $\mathbb{R}^{2 n+1}$ with center $w_{0}$ and radius $r$. Here, $d_{c}$ is the Carnot-Carathéodory distance (or control distance) generated by the vector fields $(1.3)$ (see $[11,34]$ ). We define the distance from the origin on $\mathbb{R}^{2 n+1}$ by

$$
\rho=\rho(w)=\left(|z|^{4 k}+l^{2}\right)^{1 / 4 k}
$$

which is homogeneous of degree one with respect to the natural dilation

$$
\delta_{\tau}(z, l)=\left(\tau z, \tau^{2 k} l\right), \quad \tau>0,(z, l) \in \mathbb{R}^{2 n+1} .
$$

The function $\rho$ is related to the fundamental solution of subelliptic $p$-Laplacian and subLaplacian $\Delta_{k}$ at the origin (see, $[2,3,4,36]$ ).

The sub-elliptic gradient is the $2 n$ dimensional vector field given by

$$
X=\left(X_{1}, \ldots, X_{2 n}\right),
$$

where $X_{j}$ and $X_{j+n}$ are the smooth vector fields which is defined by (1.3). If $\phi$ is a smooth radial function then we have the following lemma.

LeMma 2.1. Let $\phi=\phi(\rho)$ be a smooth radial function (i.e., $\phi$ only depends on the function $\rho$ in (2.1)). Then

$$
|X \phi|=\frac{|z|^{2 k-1}}{\rho^{2 k-1}}\left|\phi^{\prime}(\rho)\right| .
$$

Proof. The proof is an easy computation (see [32]).

Let

$$
B_{R}(0):=\left\{(z, l) \in \mathbb{R}^{2 n} \times \mathbb{R}: \rho<R\right\} .
$$

be the ball with respect to $\rho$ centered at the origin $(0,0) \in \mathbb{R}^{2 n} \times \mathbb{R}$ with radius $R$. Let $D=B_{R_{2}}(0) \backslash \overline{B_{R_{1}}(0)}$ be an annulus with $0 \leq R_{1}<R_{2} \leq \infty$ and $\phi \in L^{1}(D)$. In order to compute $\int_{D} \phi(w) d w$, we use the following transformation which is a modification of spherical transformation in [5] 
Let $w=(z, l)=(x, y, l)$ and

$$
\begin{aligned}
x_{1}= & \rho(\sin \varphi)^{1 / 2 k} \cos \psi_{1} \cos \theta_{1}, \\
y_{1}= & \rho(\sin \varphi)^{1 / 2 k} \cos \psi_{1} \sin \theta_{1}, \\
& \vdots \\
x_{n-1}= & \rho(\sin \varphi)^{1 / 2 k} \sin \psi_{1} \cdots \sin \psi_{n-2} \cos \psi_{n-1} \cos \theta_{n-1}, \\
y_{n-1}= & \rho(\sin \varphi)^{1 / 2 k} \sin \psi_{1} \cdots \sin \psi_{n-2} \cos \psi_{n-1} \sin \theta_{n-1}, \\
x_{n}= & \rho(\sin \varphi)^{1 / 2 k} \sin \psi_{1} \cdots \sin \psi_{n-2} \sin \psi_{n-1} \cos \theta_{n}, \\
y_{n}= & \rho(\sin \varphi)^{1 / 2 k} \sin \psi_{1} \cdots \sin \psi_{n-2} \sin \psi_{n-1} \sin \theta_{n}, \\
l= & \rho^{2 k} \cos \varphi,
\end{aligned}
$$

for $R_{1}<\rho<R_{2}, 0 \leq \varphi \leq \pi, 0 \leq \psi_{j} \leq \pi / 2, j=1, \ldots, n-1$, and $0 \leq \theta_{j} \leq 2 \pi, j=1, \ldots, n$. Then the volume element satisfies

$$
d w=d x d y d l=\rho^{Q-1} d \rho(\sin \varphi)^{n-k} d \varphi \prod_{j=1}^{n-1}\left[\cot \psi_{j}\left(\sin \psi_{j}\right)^{2(n-j)} d \psi_{j}\right] \prod_{j=1}^{n} d \theta_{j},
$$

where

$$
Q=2 k+2 n
$$

is the homogeneous dimension.

Hardy-type inequalities. The prominent role of Hardy's inequality in partial differential equation has been known since the pioneering paper of Baras and Goldstein [1]. Their results stimulated several interesting results in the study of linear and nonlinear parabolic equations with singular potential (see $[6,14,19,20,21,18,22,23,28,29,30,31,32]$ ).

We should also mention that there has been great interest in the study of sharp Hardytype inequalities on the sub-Riemanian space (see $[8,15,16,22,32,35,36])$. The following Hardy-type inequality associated with the vector fields (1.3) has been proved by Zhang and Niu [36]

Theorem 2.2. Let $\phi \in C_{0}^{\infty}\left(\mathbb{R}^{2 n+1} \backslash\{(0,0)\}\right), k>1$ and $1<p<2 k+2 n$. Then

$$
c_{p}(k, n) \int \frac{|z|^{(2 k-1) p}}{\left(|z|^{4 k}+l^{2}\right)^{p / 2}}|\phi|^{p} d z d l \leq \int|X \phi|^{p} d z d l
$$

where $c_{p}(k, n)=((2 k+2 n-p) / p)^{p}$.

Singular potentials. In this paper, we will focus on some singular potentials (since they are critical). As a concrete example, we will treat positive singular potential

$$
V(z, l)=\frac{\lambda|z|^{(2 k-1) p}}{\left(|z|^{4 k}+l^{2}\right)^{p / 2}}
$$


and highly singular, oscillatory potential

$$
V(z, l)=\frac{\lambda|z|^{(2 k-1) p}}{\left(|z|^{4 k}+l^{2}\right)^{p / 2}}+\frac{\beta|z|^{(2 k-1) p}}{\left(|z|^{4 k}+l^{2}\right)^{p / 2}} \sin \left(\frac{1}{\left(|z|^{4 k}+l^{2}\right)^{\alpha / 4 k}}\right),
$$

where $k \in \mathbb{N}, \lambda>0, \alpha>0$ and $\beta \in \mathbb{R} \backslash\{0\}$. We prove that the nonexistence of positive solutions is intimately related with the Hardy's inequality.

We are concerned with the general positive local solution of problem (1.1) and we define the general positive local solution in the following sense.

Definition 2.3. By a positive local solution continuous off of $\mathscr{K}$, we mean

(i) $\mathcal{Y}$ is a closed Lebesgue null subset of $\Omega$,

(ii) $u:[0, T) \rightarrow L^{1}(\Omega)$ is continuous for some $T>0$,

(iii) $(w, t) \rightarrow u(w, t) \in C((\Omega \backslash \mathscr{K}) \times(0, T))$,

(iv) $u(w, t)>0$ on $(\Omega \backslash \mathscr{K}) \times(0, T)$,

(v) $\lim _{t \rightarrow 0} u(\cdot, t)=u_{0}$ in the sense of distributions,

(vi) $X u \in L_{\text {loc }}^{p}(\Omega)$ and $u$ is a solution in the sense of distributions of the PDE.

Remark 2.4. If $0<a<b<T$ and $\mathscr{K}_{o}$ is a compact subset of $\Omega \backslash \mathscr{K}$, then $u(w, t) \geq \epsilon_{1}>0$ for $(w, t) \in \mathscr{K}_{o} \times[a, b]$ for some $\epsilon_{1}>0$. We can weaken (iii), (iv) to be

(iii) $^{\prime} u(w, t)$ is positive and locally bounded on $(\Omega \backslash \mathscr{K}) \times(0, T)$,

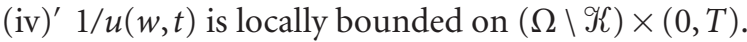

If a solution satisfies (i), (ii), (iii)', (iv)', (v), and (vi) then we call it a "general positive local solution off of $\mathscr{Y}$." This is more general than a positive local solution continuous off of $\mathscr{K}$. If $\mathscr{K}=\varnothing$, we simply call $u$ "general positive local solution."

\section{Proof of Theorem 1.1}

We argue by contradiction. Given any $T>0$, let $u:[0, T) \rightarrow L^{1}(\Omega)$ be a general positive local solution to $(1.1)$ in $(\Omega \backslash \mathscr{K}) \times(0, T)$ with $u_{0} \geq 0$ but not identically zero. Multiply both sides of (1.1) by the test function $|\phi|^{p / u^{p-1}}$ where $\phi \in C_{c}^{\infty}(\Omega \backslash \mathscr{K})$, and integrate over $\Omega$, to get

$$
\frac{1}{2-p} \frac{d}{d t} \int_{\Omega} u^{2-p}|\phi|^{p} d x-\int_{\Omega} \mathscr{L} u\left(\frac{|\phi|^{p}}{u^{p-1}}\right) d w=\int_{\Omega} V|\phi|^{p} d w .
$$

It follows from the integration by parts that

$$
\int_{\Omega} \mathscr{L} u\left(\frac{|\phi|^{p}}{u^{p-1}}\right) d w=-\int_{\Omega}|X u|^{p-2} X u \cdot X\left(\frac{|\phi|^{p}}{u^{p-1}}\right) d w .
$$

Since

$$
\begin{gathered}
|X u|^{p-2} X u \cdot X\left(\frac{|\phi|^{p}}{u^{p-1}}\right)=p|X u|^{p-2} \frac{|\phi|^{p-1}}{u^{p-1}} X u \cdot X|\phi|-(p-1) \frac{|\phi|^{p}}{u^{p}}|X u|^{p}, \\
\int_{\Omega} \mathscr{L} u\left(\frac{|\phi|^{p}}{u^{p-1}}\right) d w=(p-1) \int_{\Omega}|X u|^{p} \frac{\phi^{p}}{u^{p}} d w-p \int_{\Omega}|X u|^{p-2} \frac{\phi^{p-1}}{u^{p-1}} X u \cdot X|\phi| d w,
\end{gathered}
$$


612 Nonlinear degenerate parabolic equations

and then we have

$$
\int_{\Omega} \mathscr{L} u\left(\frac{|\phi|^{p}}{u^{p-1}}\right) d w \geq(p-1) \int_{\Omega}|X u|^{p} \frac{|\phi|^{p}}{u^{p}} d w-p \int_{\Omega}|X u|^{p-1}|X \phi| \frac{\phi^{p-1}}{u^{p-1}} d w .
$$

Here we can use the following elementary inequality: Let $p>1$ and $s_{1} \neq s_{2}$ be two positive real numbers. Then

$$
s_{1}^{p}-s_{2}^{p}-p s_{2}^{p-1}\left(s_{1}-s_{2}\right)>0
$$

it follows that

$$
(p-1) s_{2}^{p}-p s_{2}^{p-1} s_{1}>-s_{1}^{p} .
$$

We can take $s_{2}=|(\phi / u) X u|, s_{1}=|X \phi|$; then we have

$$
(p-1) \int_{\Omega}|X u|^{p} \frac{|\phi|^{p}}{u^{p}} d w-p \int_{\Omega}|X u|^{p-1}|X \phi| \frac{|\phi|^{p-1}}{u^{p-1}} d w \geq-\int_{\Omega}|X \phi|^{p} d w .
$$

Therefore

$$
\int_{\Omega} \mathscr{L} u\left(\frac{|\phi|^{p}}{u^{p-1}}\right) d w \geq-\int_{\Omega}|X \phi|^{p} d w
$$

Substituting (3.8) into (3.1) and integrating from $t_{1}$ to $t_{2}$, where $0<t_{1}<t_{2}<T$, we obtain

$$
\int_{\Omega} V(w)|\phi|^{p} d w-\int_{\Omega}|X \phi|^{p} d w \leq \frac{1}{(2-p)\left(t_{2}-t_{1}\right)} \int_{\Omega}\left(u^{2-p}\left(w, t_{2}\right)-u^{2-p}\left(w, t_{1}\right)\right)|\phi|^{p} d w .
$$

Using Jensen's inequality for concave functions, we obtain

$$
\int_{\Omega}\left(u^{(2-p)}\left(w, t_{i}\right)\right)^{(2 k+2 n) / p} d w \leq C(|\Omega|)\left(\int_{\Omega} u\left(w, t_{i}\right) d w\right)^{(2-p)(2 k+2 n) / p}<\infty
$$

Here we use the fact that $\Omega$ is bounded, whence $|\Omega|$ is finite. Therefore

$$
u^{2-p}\left(w, t_{i}\right) \in L^{(2 k+2 n) / p}(\Omega) .
$$

We now use the following a priori inequality which is a consequence of the SobolevPoincaré inequality $[7,33]$. For every $\epsilon>0$ there exists $C(\epsilon)$ such that

$$
\begin{aligned}
\frac{1}{(2-p)\left(t_{2}-t_{1}\right)} \int_{\Omega} & \left(u^{2-p}\left(w, t_{2}\right)-u^{2-p}\left(w, t_{1}\right)\right)|\phi|^{p} d w \\
& \leq \frac{\epsilon}{1-\epsilon} \int_{\Omega}|X \phi|^{p} d w+C(\epsilon) \int_{\Omega}|\phi|^{p} d w .
\end{aligned}
$$


Substituting (3.12) into (3.9), we obtain

$$
\int_{\Omega} V(w)|\phi|^{p} d w-\int_{\Omega}|X \phi|^{p} d w \leq \frac{\epsilon}{1-\epsilon} \int_{\Omega}|X \phi|^{p} d w+C(\epsilon) \int_{\Omega}|\phi|^{p} d w .
$$

Therefore

$$
\inf _{0 \neq \phi \in C_{c}^{\infty}(\Omega \mid \mathscr{K})} \frac{\int_{\Omega}|X \phi|^{p} d w-\int_{\Omega}(1-\epsilon) V(w)|\phi|^{p} d w}{\int_{\Omega}|\phi|^{p} d w} \geq-(1-\epsilon) C(\epsilon)>-\infty .
$$

This contradicts our assumption. The proof of Theorem 1.1 is now complete.

Corollary 3.1. Let $0 \in \Omega, c_{p}(k, n)=((2 k+2 n-p) / p)^{p}$ and $V(z, l)=\lambda|z|^{(2 k-1) p /\left(|z|^{4 k}+\right.}$ $\left.l^{2}\right)^{p / 2}$. Then problem (1.1) has no general positive local solution off of $\mathscr{K}$ if $\lambda>c_{p}(k, n)$ and $(4 k+4 n) /(2 k+2 n+1) \leq p<2$.

Proof. Given $\epsilon>0$, we define the radial function, $\phi \in C_{c}^{1}(\Omega) \cap W^{1, \infty}(\Omega)$, by

$$
\phi(\rho)= \begin{cases}\epsilon^{-((2 k+2 n-p) / p)} & \text { if } 0 \leq \rho \leq \epsilon, \\ \rho^{-((2 k+2 n-p) / p)} & \text { if } \epsilon \leq \rho \leq 1, \\ 2-\rho & \text { if } 1 \leq \rho \leq 2, \\ 0 & \text { if } \rho \geq 2 .\end{cases}
$$

We are assuming that $0 \in \Omega$. Without loss of generality we assume that $B_{2}(0)=\{(z, l) \in$ $\left.\mathbb{R}^{2 n} \times \mathbb{R}: \rho<2\right\} \subset \Omega$; if not, we simply redefine $\phi$, replacing 2 by $R$ where $B_{R}(0) \subset \Omega$. This only results in notational changes in the proof that follows. Then we assume (without loss of generality) that $\phi \in C_{c}^{\infty}(\Omega)$.

We want to show that

$$
\inf _{0 \neq \phi \in C_{c}^{\infty}(\Omega \mid \mathscr{K})} \frac{\int_{\Omega}|X \phi|^{p} d z d l-\int_{\Omega}\left(\lambda|z|^{\left.(2 k-1) p /\left(|z|^{4 k}+l^{2}\right)^{p / 2}\right)|\phi|^{2} d z d l}\right.}{\int_{\Omega}|\phi|^{p} d w}=-\infty .
$$

Using Lemma 2.1, we get, for $\phi$ as in (3.15),

$$
|X \phi(\rho)|^{p}= \begin{cases}0 & \text { if } 0 \leq \rho<\epsilon, \\ \left(\frac{2 k+2 n-p}{p}\right)^{p} \rho^{-2 k-2 n\left(\frac{|z|}{\rho}\right)^{(2 k-1) p}} & \text { if } \epsilon<\rho<1, \\ \left(\frac{|z|}{\rho}\right)^{(2 k-1) p} & \text { if } 1<\rho<2, \\ 0 & \text { if } \rho>2 .\end{cases}
$$


614 Nonlinear degenerate parabolic equations

Using $|z|=\rho(\sin \varphi)^{1 / 2 k}$ then we obtain

$$
\begin{aligned}
\int_{\Omega}|X \phi|^{p} d w & =\mu\left[\int_{\epsilon}^{1}\left(\frac{2 k+2 n-p}{p}\right)^{p} \frac{d \rho}{\rho}+\int_{1}^{2} \rho^{2 k+2 n-1} d \rho\right] \\
& =\mu\left[-\left(\frac{2 k+2 n-p}{p}\right)^{p} \log \epsilon+\frac{2^{2 k+2 n}-1}{2 k+2 n}\right],
\end{aligned}
$$

where

$$
\mu=\int_{0}^{\pi}(\sin \varphi)^{n-k+(2 k-1) p / 2 k} d \varphi \times \int_{0}^{\pi / 2} \prod_{j=1}^{n-1}\left[\cot \psi_{j}\left(\sin \psi_{j}\right)^{2(n-j)} d \psi_{j}\right] \times \int_{0}^{2 \pi} \prod_{j=1}^{n} d \theta_{j}
$$

Since

$$
V(z, l)=\frac{\lambda|z|^{(2 k-1) p}}{\left(|z|^{4 k}+l^{2}\right)^{p / 2}}=\frac{\lambda}{\rho^{p}}(\sin \varphi)^{(2 k-1) p / 2 k},
$$

we write

$$
\begin{aligned}
& \int_{\Omega} \frac{\lambda|z|^{(2 k-1) p}}{\left(|z|^{4 k}+l^{2}\right)^{p / 2}}|\phi|^{p} d w \\
& \quad=\lambda \mu\left[\epsilon^{p-2 k-2 n} \int_{0}^{\epsilon} \rho^{2 k+2 n-p-1} d \rho+\int_{\epsilon}^{1} \frac{1}{\rho} d \rho+\int_{1}^{2}(2-\rho)^{p} \rho^{2 k+2 n-p-1} d \rho\right] \\
& \quad=\lambda \mu\left(-\log \epsilon+\frac{1}{2 k+2 n-p}+\frac{\xi^{2 k+2 n-p}-1}{2 k+2 n-p}\right)
\end{aligned}
$$

where $\xi \in(1,2)$. Note that we applied the Generalized Second Mean Value Theorem for integrals to the last integral above.

Next,

$$
\begin{aligned}
\int_{\Omega}|\phi|^{p}(w) d w & =\gamma\left[\epsilon^{p-2 k-2 n} \int_{0}^{\epsilon} \rho^{2 k+2 n-1} d \rho+\int_{\epsilon}^{1} \rho^{p-1} d \rho+\int_{1}^{2}(2-\rho)^{p} \rho^{2 k+2 n-1} d \rho\right] \\
& =\gamma\left[\frac{\epsilon^{p}}{2 k+2 n}+\frac{1-\epsilon^{p}}{p}+\frac{\eta^{2 k+2 n}-1}{2 k+2 n}\right] \\
& =\gamma\left[\frac{1}{p}+\frac{\eta^{2 k+2 n}-1}{2 k+2 n}\right](1+o(1)) \quad \text { as } \epsilon \longrightarrow 0,
\end{aligned}
$$

where $\eta \in(1,2)$ and

$$
\gamma=\int_{0}^{\pi}(\sin \varphi)^{n-k} d \varphi \times \int_{0}^{\pi / 2} \prod_{j=1}^{n-1}\left[\cot \psi_{j}\left(\sin \psi_{j}\right)^{2(n-j)} d \psi_{j}\right] \times \int_{0}^{2 \pi} \prod_{j=1}^{n} d \theta_{j} .
$$


Substituting (3.18), (3.21) and (3.22) into the Rayleigh quotient,

$$
\begin{aligned}
\mathscr{R} & =\frac{\int_{\Omega}|X \phi|^{p} d z d l-\int_{\Omega} \frac{\lambda|z|^{(2 k-1) p}}{\left(|z|^{4 k}+l^{2}\right)^{p / 2}}|\phi|^{p} d z d l}{\int_{\Omega}|\phi|^{p} d z d l} \\
& =\frac{\mu\left[-\left(\frac{2 k+2 n-p}{p}\right)^{p} \log \epsilon+\frac{2^{2 k+2 n}-1}{2 k+2 n}-\lambda\left(-\log \epsilon+\frac{1}{2 k+2 n-p}+\frac{\xi^{2 k+2 n-p}-1}{2 k+2 n-p}\right)\right]}{\gamma\left[\frac{1}{p}+\frac{\eta^{2 k+2 n}-1}{2 k+2 n}\right](1+o(1))} \\
& =\frac{\mu\left[\left(\lambda-\left(\frac{2 k+2 n-p}{p}\right)^{p}\right) \log \epsilon+\frac{2^{2 k+2 n}-1}{2 k+2 n}-\lambda\left(\frac{1}{2 k+2 n-p}+\frac{\xi^{2 k+2 n-p}-1}{2 k+2 n-p}\right)\right]}{\gamma\left[\frac{1}{p}+\frac{\eta^{2 k+2 n}-1}{2 k+2 n}\right](1+o(1))} .
\end{aligned}
$$

Therefore taking limits as $\epsilon \rightarrow 0^{+}$, we find that the right-hand side of (3.24) can become negative and arbitrarily large in magnitude, that is,

$$
\inf _{0 \neq \phi \in(\Omega \mid \mathcal{K})} \frac{\int_{\Omega}|X \phi|^{p} d z d l-\int_{\Omega}\left(\lambda|z|^{(2 k-1) p} /\left(|z|^{4 k}+l^{2}\right)^{p / 2}\right)|\phi|^{p} d z d l}{\int_{\Omega}|\phi|^{p} d z d l}=-\infty
$$

The proof of Corollary 3.1 is now complete.

Corollary 3.2. Let $0 \in \Omega, c_{p}(k, n)=((2 k+2 n-p) / p)^{p}$ and $V(z, l)$ defined by (2.11). Then problem (1.1) has no general positive local solution off of $\mathscr{K}$ if $\lambda>c_{p}(k, n)$ and $(4 k+$ $4 n) /(2 k+2 n+1) \leq p<2$.

Proof. The proof of Corollary 3.2 is similar and we omit the details. Although oscillating part of $V(z, l)$ is so singular at the origin, nonexistence of positive solutions only depends on the size of $\lambda$. The point is that oscillating part of the potential has very large positive and negative parts, in particular, it oscillates wildly, but important cancellations occur between the positive and the negative parts in the quadratic form.

\section{References}

[1] P. Baras and J. A. Goldstein, The heat equation with a singular potential, Trans. Amer. Math. Soc. 284 (1984), no. 1, 121-139.

[2] R. Beals, B. Gaveau, and P. Greiner, On a geometric formula for the fundamental solution of subelliptic Laplacians, Math. Nachr. 181 (1996), 81-163.

[3] _ _ Uniforms hypoelliptic Green's functions, J. Math. Pures Appl. (9) 77 (1998), no. 3, 209248.

[4] Green's functions for some highly degenerate elliptic operators, J. Funct. Anal. 165 (1999), no. 2, 407-429.

[5] R. Beals, B. Gaveau, P. Greiner, and J. Vauthier, The Laguerre calculus on the Heisenberg group. II, Bull. Sci. Math. (2) 110 (1986), no. 3, 225-288.

[6] X. Cabré and Y. Martel, Existence versus explosion instantanée pour des équations de la chaleur linéaires avec potentiel singulier [Existence versus instantaneous blowup for linear heat equations with singular potentials], C. R. Acad. Sci. Paris Sér. I Math. 329 (1999), no. 11, 973-978 (French).

[7] L. Capogna, D. Danielli, and N. Garofalo, An embedding theorem and the Harnack inequality for nonlinear subelliptic equations, Comm. Partial Differential Equations 18 (1993), no. 9-10, 1765-1794. 
[8] L. D'Ambrosio, Hardy inequalities related to Grushin type operators, Proc. Amer. Math. Soc. 132 (2004), no. 3, 725-734.

[9] A. El Hamidi and M. Kirane, Nonexistence results of solutions to systems of semilinear differential inequalities on the Heisenberg group, Abstr. Appl. Anal. 2004 (2004), no. 2, 155-164.

[10] A. El Hamidi and A. Obeid, Systems of semilinear higher-order evolution inequalities on the Heisenberg group, J. Math. Anal. Appl. 280 (2003), no. 1, 77-90.

[11] C. Fefferman and D. H. Phong, Subelliptic eigenvalue problems, Conference on Harmonic Analysis in Honor of Antoni Zygmund, Vol. I, II (Chicago, Ill, 1981) (W. Beckner, A. P. Calderón, R. Efferman, and P. W. Jones, eds.), Wadsworth Math. Ser., Wadsworth, California, 1983, pp. 590-606.

[12] G. B. Folland, A fundamental solution for a subelliptic operator, Bull. Amer. Math. Soc. 79 (1973), no. 2, 373-376.

[13] Subelliptic estimates and function spaces on nilpotent Lie groups, Ark. Mat. 13 (1975), no. 2, 161-207.

[14] J. P. García Azorero and I. Peral Alonso, Hardy inequalities and some critical elliptic and parabolic problems, J. Differential Equations 144 (1998), no. 2, 441-476.

[15] N. Garofalo, Unique continuation for a class of elliptic operators which degenerate on a manifold of arbitrary codimension, J. Differential Equations 104 (1993), no. 1, 117-146.

[16] N. Garofalo and E. Lanconelli, Frequency functions on the Heisenberg group, the uncertainty principle and unique continuation, Ann. Inst. Fourier (Grenoble) 40 (1990), no. 2, 313-356.

[17]__ Existence and nonexistence results for semilinear equations on the Heisenberg group, Indiana Univ. Math. J. 41 (1992), no. 1, 71-98.

[18] G. R. Goldstein, J. A. Goldstein, and I. Kombe, Nonlinear parabolic equations with singular coefficient and critical exponent, to appear in Appl. Anal.

[19] J. A. Goldstein and I. Kombe, Instantaneous blow up, Advances in Differential Equations and Mathematical Physics (Birmingham, Ala, 2002), Contemp. Math., vol. 327, American Mathematical Society, Rhode Island, 2003, pp. 141-150.

[20] _ Nonlinear degenerate parabolic equations with singular lower-order term, Adv. Differential Equations 8 (2003), no. 10, 1153-1192.

[21] Nonlinear degenerate parabolic equations on the Heisenberg group, Int. J. Evol. Equ. 1 (2005), no. 1, 1-22.

[22] J. A. Goldstein and Q. S. Zhang, On a degenerate heat equation with a singular potential, J. Funct. Anal. 186 (2001), no. 2, 342-359.

[23] Linear parabolic equations with strong singular potentials, Trans. Amer. Math. Soc. 355 (2003), no. 1, 197-211.

[24] P. C. Greiner, A fundamental solution for a nonelliptic partial differential operator, Canad. J. Math. 31 (1979), no. 5, 1107-1120.

[25] L. Hörmander, Hypoelliptic second order differential equations, Acta Math. 119 (1967), 147171.

[26] D. S. Jerison and J. M. Lee, Extremals for the Sobolev inequality on the Heisenberg group and the CR Yamabe problem, J. Amer. Math. Soc. 1 (1988), no. 1, 1-13.

[27] , Intrinsic CR normal coordinates and the CR Yamabe problem, J. Differential Geom. 29 (1989), no. 2, 303-343.

[28] I. Kombe, Doubly nonlinear parabolic equations with singular lower order term, Nonlinear Anal. 56 (2004), no. 2, 185-199.

[29] - The linear heat equation with highly oscillating potential, Proc. Amer. Math. Soc. 132 (2004), no. 9, 2683-2691.

[30] Nonlinear parabolic partial differential equations for Baouendi-Grushin operator, to appear in Mathematische Nachrichten. 
[31] , On the nonexistence of positive solutions to Doubly nonlinear equations for BaouendiGrushin operator, preprint 2005.

[32] On the nonexistence of positive solutions to nonlinear degenerate parabolic equations with singular coefficients, to appear in Appl. Anal.

[33] G. Lu, The sharp Poincaré inequality for free vector fields: an endpoint result, Rev. Mat. Iberoamericana 10 (1994), no. 2, 453-466.

[34] A. Nagel, E. M. Stein, and S. Wainger, Balls and metrics defined by vector fields. I. Basic properties, Acta Math. 155 (1985), no. 1-2, 103-147.

[35] P. Niu, H. Zhang, and Y. Wang, Hardy type and Rellich type inequalities on the Heisenberg group, Proc. Amer. Math. Soc. 129 (2001), no. 12, 3623-3630.

[36] H. Zhang and P. Niu, Hardy-type inequalities and Pohozaev-type identities for a class of pdegenerate subelliptic operators and applications, Nonlinear Anal. 54 (2003), no. 1, 165-186.

Ismail Kombe: Mathematics Department, Dawson-Loeffler Science and Mathematics Building, Oklahoma City University, 2501 North Blackwelder, Oklahoma City, OK 73106-1493, USA

E-mail address: ikombe@okcu.edu 


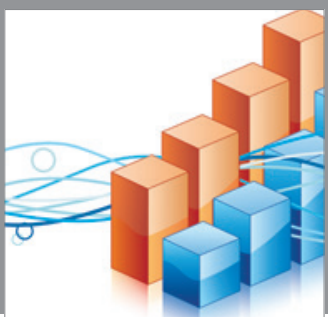

Advances in

Operations Research

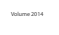

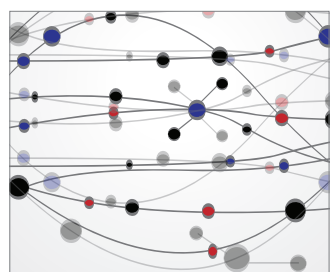

\section{The Scientific} World Journal
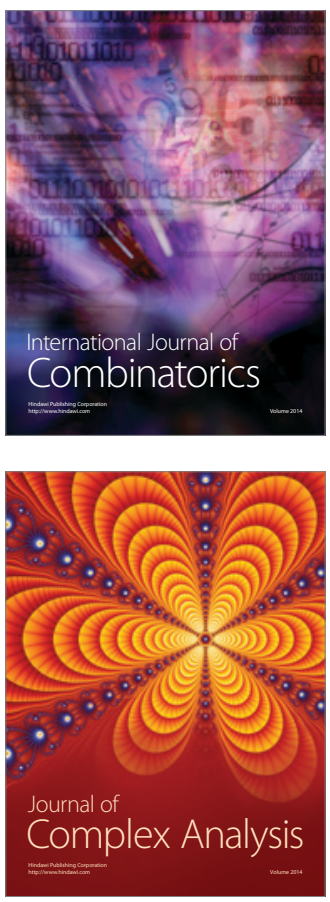

International Journal of

Mathematics and

Mathematical

Sciences
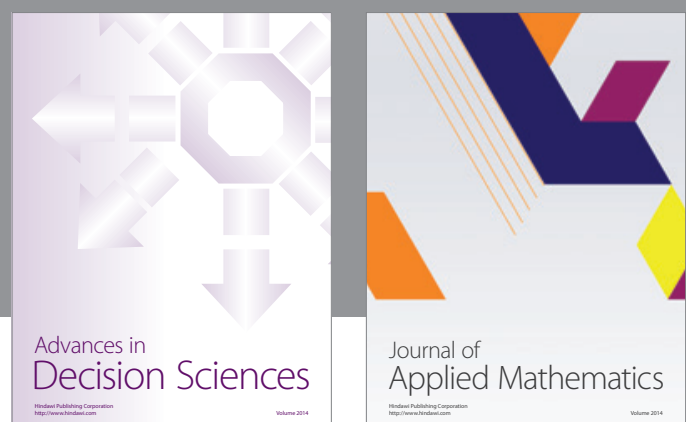

Journal of

Applied Mathematics
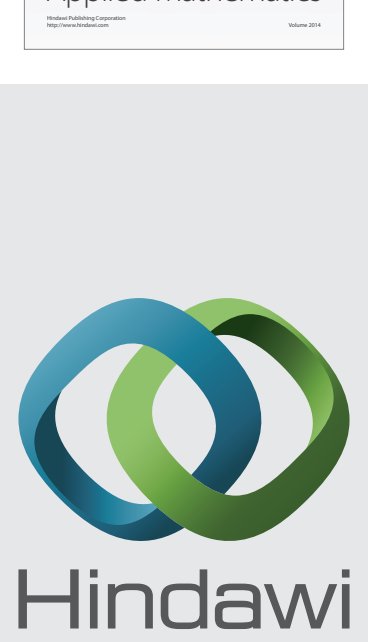

Submit your manuscripts at http://www.hindawi.com
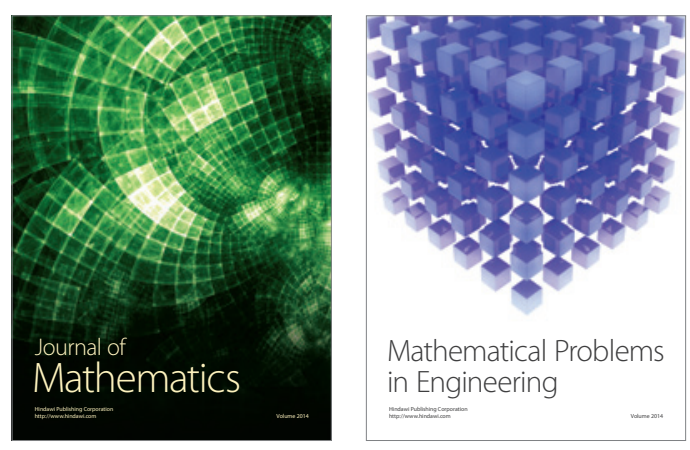

Mathematical Problems in Engineering
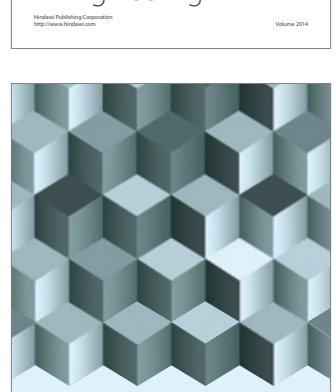

Journal of

Function Spaces
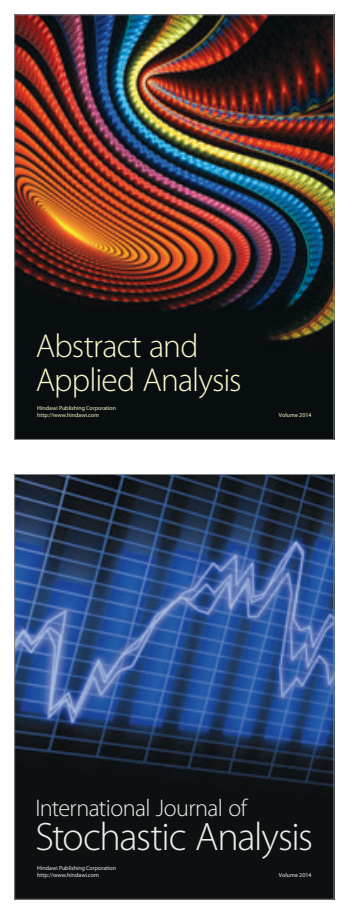

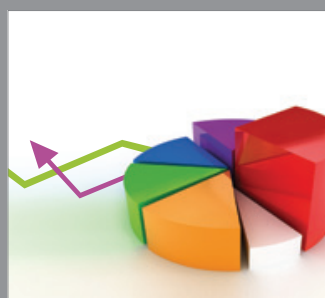

ournal of

Probability and Statistics

Promensencen
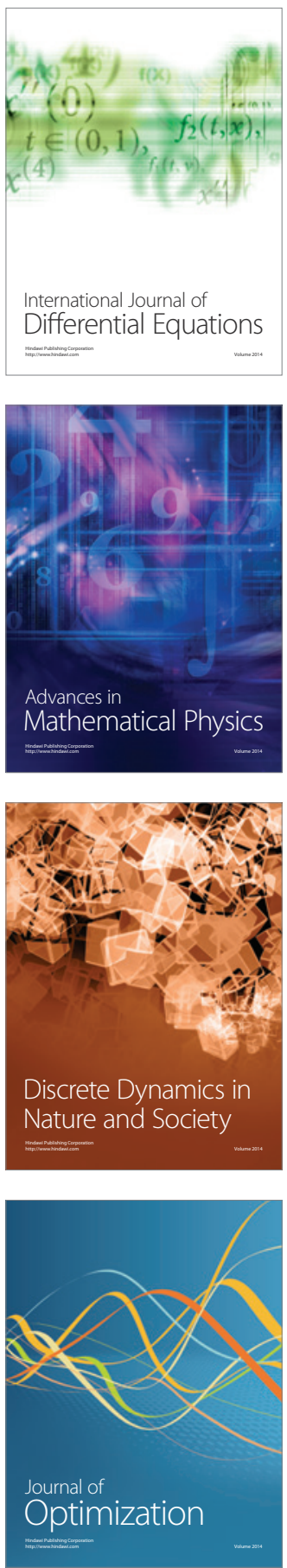